\title{
Exact Outage Probability of Cooperative Diversity with Opportunistic Spectrum Access
}

\author{
Himal A. Suraweera*, Peter J. Smith ${ }^{\dagger}$ and Nusrat A. Surobhi* \\ * School of Electrical Engineering, Victoria University \\ PO Box 14428, Melbourne, Victoria 8001, Australia \\ ${ }^{\dagger}$ Department of Electrical and Computer Engineering, University of Canterbury \\ Private Bag 4800, Christchurch, New Zealand \\ Email: himal.suraweera@vu.edu.au,p.smith@elec.canterbury.ac.nz,nusrat.ahmedsurobhi@ research.vu.edu.au
}

\begin{abstract}
Opportunistic spectrum access, popularly known as cognitive radio technology is an innovative radio design philosophy which aims to increase spectrum utilization by exploiting unused spectrum in dynamic environments. In this paper, we present an exact outage performance analysis for the rates of a decode-and-forward cooperative network where a source communicates with its destination using the wellknown repetition-based relaying scheme or using the single best relay, i.e, selection cooperation. Closed-form expressions are obtained for independent Rayleigh fading channels. Selection cooperation exhibits lower outage probabilities compared to the repetition-based scheme. However, the inability of relays to sense unoccupied spectrum can cause a larger drop in the outage performance of selection cooperation, especially in the high SNR regime. The analytical outage probability expressions have been validated through simulations.
\end{abstract}

\section{INTRODUCTION}

Radio spectrum is one of the most scarce and valuable resources for wireless communications. Strictly conservative spectrum policies employed by regulatory authorities have resulted in underutilization of the overall available spectrum. Measurements performed by, for example, the Federal Communications Commission (FCC) [1] in the United States and Ofcom [2] in the United Kingdom have revealed that at any given time, a large number of spectrum bands are seldom or rarely occupied.

Cognitive radio is a promising technology to exploit such spectrum "white spaces" and has received significant interest in the research community [3], [4]. Cognitive radios can coexist with primary users and access parts of the spectrum for information transmission, provided that they cause minimal interference to the primary users operating in that band.

The complementary technique of cooperative diversity is a promising technique for providing the high data-rate coverage required in future cellular and ad-hoc wireless communication networks [5]. In cooperative relaying, one or more intermediate nodes are used to support signal transmission when the direct path from source to destination is in a deep fade. Relays can provide transmit power savings due to path loss reduction and spatial diversity.

This research was supported by a Discovery Grant (DP0774689) from the Australian Research Council.
Recently, the combination of relaying and cognitive radio techniques has also been investigated [6], [7], [9], [10]. In addition to the advantages described above, cognitive relays could also be deployed as a means of minimizing the interference caused by secondary transmissions to the primary licensee, while guaranteeing reliable communications for the secondary users [9]. An ad-hoc cognitive radio concept, in which transceivers with small power and multi-hop communications are used for expanding the service area has been proposed in [6]. In [10], several distributed transmit power allocation schemes for relay-based cognitive radio systems utilizing "gray space" spectrum have been developed. Outage probability and average error performance are widely used measures for characterizing the performance of communication systems [11], [12]. In [7], Lee and Yener have studied the approximate outage probability of a repetitionbased $^{1}$ cognitive relay network in the high signal-to-noise ratio (SNR) regime. Their main emphasis was to analyse the achievable diversity order of the system subject to cognitive relays being able to acquire spectrum. In contrast to the standard relay scenario, cognitive relays will only transmit if they are successful in obtaining spectrum (this is the case even if they correctly decode the source information). It was shown in [7] that under imperfect spectrum acquisition, full diversity cannot be realized.

Practical systems often operate in the low to medium SNR regimes. Hence, it is likely that system design will be for relatively high rate outage probability values [14]. While asymptotic (high SNR) performance analysis highlights the diversity order achievable by various techniques, it is also important to study exact performance in the finite SNR regime, so as to compare various schemes in practical settings [11], [14]. Building on the work in [7], in this paper we investigate the exact outage probability valid at all SNRs for a decodeand-forward cognitive relay network. We compare the outage performance of the repetition-based scheme with selection cooperation. Under imperfect spectrum acquisition scenarios, the outage probability of both schemes degrades significantly.

\footnotetext{
${ }^{1}$ Throughout the paper, we use the terminology adopted in [8] and refer to the relaying protocol in which a time-division channel allocation arrangement is used to facilitate orthogonal transmission as "repetition-based relaying".
} 
However, compared to the repetition-based scheme, failure to sense unoccupied spectrum at the relays causes a larger drop in the outage performance of selection cooperation, especially in the high SNR regime.

This paper is organized as follows: Section II presents the relay system, channel model and details of the spectrum acquisition process. In Section III, the exact outage probability of the two cooperative diversity protocols are investigated. Numerical and simulation results are presented in Section IV and finally some conclusions appear in Section V.

\section{Cognitive Relay Network}

\section{A. System Model}

We consider the same cognitive relay network architecture described in $[7$, Sec. II]. The source transmission to the destination is assisted by several cognitive relay nodes. In this paper, in addition to the repetition-based relaying scheme considered in [7], we also investigate the outage probability of the selection cooperation scheme. In phase I, both the relays and the destination receive the message transmitted by the source, as shown in Fig. 1. In phase II, the two schemes behave differently. In the repetition-based scheme, all relays participate in phase II in a time-division arrangement. With selection cooperation [13], [14] only a single relay node (the relay with the best instantaneous relay-destination channel) in the decoding set, $\mathcal{R}(s)$, is used to forward information to the destination. Furthermore, in contrast to the relay functionality assumed in [8], [11], [12], the cognitive relays can only retransmit information if they are successful in obtaining spectrum during the spectrum sensing period. Therefore, in some cases, even if cognitive relay(s) are able to decode the data from the source successfully they might not be able to assist in relaying due to the unavailability of spectrum.

\section{B. Channel Model}

The channels between nodes $i$ and $j$ are modelled as independent slowly varying flat Rayleigh fading random variables (RVs) with variance $1 / \lambda_{i, j}$. Hence, $X=\left|h_{i, j}\right|^{2}$ is an exponentially distributed random variable with probability density function (PDF), $f_{X}(x)=\lambda_{i, j} \exp \left(-\lambda_{i, j} x\right)$. There are two important cases here. In the main body of the paper we assume for simplicity that $\lambda_{i, j}=\lambda_{s r}$ for all source-relay channels and $\lambda_{i, j}=\lambda_{r d}$ for all relay-destination channels. The general case where all the $\lambda_{i, j}$ parameters are different is shown in Appendix B.

\section{Spectrum Acquisition}

The reliability of spectrum acquisition depends heavily on the sensing mechanism employed. To this end, costly high sensitivity sensing modules could be added to the relay hardware. However, energy detection is a simple technique for detecting an unknown signal in the presence of AWGN and this is the approach considered here. The aim of spectrum sensing is to decide between the hypotheses $\mathcal{H}_{0}$ and $\mathcal{H}_{1}$ :

$$
Y_{p r}(t)= \begin{cases}N_{p r}(t), & \mathcal{H}_{0} \\ h_{p r} X_{p}(t)+N_{p r}(t), & \mathcal{H}_{1}\end{cases}
$$

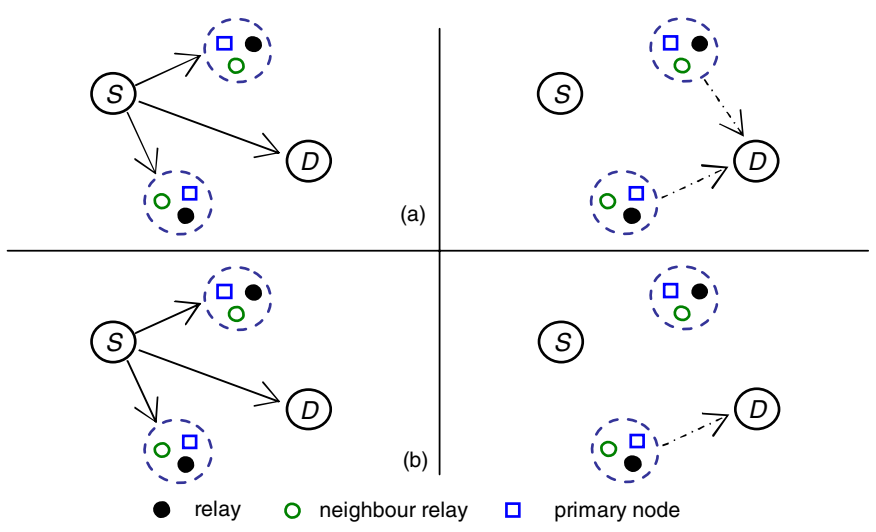

Fig. 1. Phase I and II transmissions of (a) repetition-based scheme and (b) selection cooperation.

where $X_{p}(t)$ is primary user's transmitted signal, $N_{p r}(t)$ is the AWGN component at the relay and $h_{p r}$ is the amplitude gain of the primary-relay channel. At the relay, $Y_{p r}(t)$ is bandwidth limited and a squaring device is used to measure the received energy. The average probability of detection, $P_{d}$, in a Rayleigh fading environment is given by

$$
\begin{aligned}
P_{d} & =e^{-\zeta_{\tau} / 2} \sum_{n=0}^{u-2} \frac{1}{n !}\left(\frac{\zeta_{\tau}}{2}\right)^{n}+\left(\frac{1+\bar{\rho}}{\bar{\rho}}\right)^{u-1} \\
& \times\left[e^{-\frac{\zeta_{\tau}}{2(1+\bar{\rho})}}-e^{\zeta_{\tau} / 2} \sum_{n=0}^{u-2} \frac{1}{n !}\left(\frac{\zeta_{\tau} \bar{\rho}}{2(1+\bar{\rho})}\right)^{n}\right],
\end{aligned}
$$

where $u$ is the time bandwidth product, $\bar{\rho}$ is the average SNR and $\zeta_{\tau}$ is the decision threshold employed to decide whether the primary signal is present or not [16]. The probability of a false-alarm, $P_{f}$, is given by

$$
P_{f}=Q\left(u, \zeta_{\tau} / 2\right) \text {. }
$$

In (3), $Q(\cdot, \cdot)$ is the regularized incomplete gamma function defined as $Q(a, x)=1 / \Gamma(a) \int_{x}^{\infty} t^{a-1} e^{-t} d t$. Another effective solution to increase the reliability of spectrum sensing is to employ a cooperative spectrum sensing protocol [16]. If cooperative spectrum sensing (i.e., $N$ relay nodes including the potential relay conduct joint spectrum sensing) and the OR-rule for deciding in favor of the presence of a spectrum hole is employed, the probabilities of detection and false alarm are given by

$$
C_{d}=1-\left(1-P_{d}\right)^{N+1}, \quad C_{f}=1-\left(1-P_{f}\right)^{N+1} .
$$

\section{Outage Probability}

In this section, outage probabilities of the repetition-based scheme and the selection cooperation scheme are derived for both perfect and imperfect spectrum acquisition.

\section{A. Repetition-based Relaying Scheme}

First, consider the idealistic scenario where all potential relays are capable of acquiring the spectrum for their transmissions to the destination. 
The mutual information between the source and cooperative relay nodes, $c=1, \ldots, m$, is given by

$$
I_{c}=\frac{1}{m+1} \log _{2}\left(1+\mathrm{SNR}\left|h_{s c}\right|^{2}\right),
$$

where SNR is the transmitted SNR and $m$ is the total number of clusters. The availability of a relay node to assist the source to destination communication, depends on the reliability of that source to relay transmission link. That is, when the mutual information of a source-relay link becomes greater than the target rate, $R$, the node joins the cooperative set, $c \in \mathcal{R}(s)$. Hence, the mutual information of the repetition-based relaying scheme is expressed as

$$
I=\frac{1}{m+1} \log _{2}\left(1+\operatorname{SNR}\left(\left|h_{s d}\right|^{2}+\sum_{\mathcal{R}(s)}\left|h_{c d}\right|^{2}\right)\right) .
$$

Outage probability is one of the most commonly used performance measures in wireless systems and is defined as $P_{\text {out }}=\operatorname{Pr}(I<R)$. Using the law of total probability, $P_{\text {out }}$ can be written as [11]

$$
P_{\text {out }}=\sum_{\mathcal{R}(s)} \operatorname{Pr}[I<R \mid \mathcal{R}(s)] \operatorname{Pr}[\mathcal{R}(s)] .
$$

Note that $\operatorname{Pr}[I<R|| \mathcal{R}(s) \mid=1, \ldots, k]$ is given by

$$
\operatorname{Pr}\left(\left|h_{s d}\right|^{2}+\sum_{c=1}^{k}\left|h_{r_{c} d}\right|^{2}<\frac{2^{(m+1) R}-1}{\mathrm{SNR}}\right) .
$$

Define the terms in (8) by $\gamma=\frac{2^{(m+1) R}-1}{\mathrm{SNR}}, \mathcal{X}_{1}=\left|h_{s, d}\right|^{2}$ and $\mathcal{X}_{2}=\sum_{c=1}^{k}\left|h_{r_{c}, d}\right|^{2}$. It is well known that the PDF of $\mathcal{X}_{2}$ is a gamma PDF with shape parameter $k$ and scale parameter $\lambda_{r d}$. The PDF, $p_{\mathcal{X}}(x)$, of $\mathcal{X}=\mathcal{X}_{1}+\mathcal{X}_{2}$ is given by [17]

$$
\begin{aligned}
p_{\mathcal{X}}(x) & =\lambda_{s d} \lambda_{r d}^{k}\left(\frac{1}{\left(\lambda_{s d}-\lambda_{r d}\right)^{k}} e^{-\lambda_{s d} x}\right. \\
& \left.-\left(\sum_{j=1}^{k} \frac{1}{(j-1) !} x^{j-1}\left(\lambda_{r d}-\lambda_{s d}\right)^{j-k-1}\right) e^{-\lambda_{r d} x}\right) .
\end{aligned}
$$

Using [18, eq. (3.381-1)], the cumulative distribution function (CDF) $F_{\mathcal{X}}(x)=\int_{0}^{x} p_{\mathcal{X}}(u) d u$ can be expressed as (10). Note, that we have used an alternative representation for the lower incomplete gamma function $\gamma(a, x)$ where $a$ is an integer [18, eq. (8.352-1)]. Finally we note that

$$
\operatorname{Pr}[|\mathcal{R}(s)|=k]=\left(\begin{array}{c}
m \\
k
\end{array}\right)\left(e^{-\lambda_{s r} \gamma}\right)^{k}\left(1-e^{\lambda_{s r} \gamma}\right)^{m-k},
$$

and using (10) and (11) we can express $P_{\text {out }}$ for perfect spectrum acquisition as (12).

Next we consider the outage performance of the cooperative relay system under imperfect spectrum acquisition. Cognitive relays, as noted in [7], may not be able to acquire a spectrum hole successfully. Hence, even if the relays correctly decode the source transmission, they may not be able to retransmit towards the destination. This feature differentiates the relay capability of a cognitive network from that of a conventional cooperative network. The probability of each relay being in the relaying set is simply [7]

$$
\operatorname{Pr}\left[r_{c} \in \mathcal{R}(s)\right]=e^{-\lambda_{s r} \frac{2^{(m+1) R}-1}{\gamma}} P_{d},
$$

and the outage probability can be calculated by conditioning on the number of successful relays as

$$
\begin{aligned}
P_{\text {out }}=\sum_{k=0}^{m}\left(\sum_{i=0}^{k}\right. & \operatorname{Pr}[I<R \| \mathcal{R}(s) \mid=i, \mathcal{K}=k] \\
& \times \operatorname{Pr}[|R(s)|=i \mid \mathcal{K}=k]) \operatorname{Pr}[\mathcal{K}=k] .
\end{aligned}
$$

In (14), $\mathcal{K}$ is the number of successful potential relays and the probabilities $\operatorname{Pr}[|R(s)|=i \mid \mathcal{K}=k]$ and $\operatorname{Pr}[\mathcal{K}=k]$ are given by

$$
\operatorname{Pr}[|R(s)|=i \mid \mathcal{K}=k]=\left(\begin{array}{c}
k \\
i
\end{array}\right)\left(e^{-\lambda_{s r} \gamma}\right)^{i}\left(1-e^{\lambda_{s r} \gamma}\right)^{k-i}
$$

and

$$
\operatorname{Pr}[\mathcal{K}=k]=\left(\begin{array}{c}
m \\
k
\end{array}\right) P_{d}^{k}\left(1-P_{d}\right)^{m-k} .
$$

Therefore, substituting (15) and (16) in (14), the exact outage probability due to imperfect spectrum acquisition can be calculated.

\section{B. Selection Cooperation}

Selection cooperation has been shown to be an alternative to cooperative diversity schemes based on repetition [13], [14]. Recently, Beres and Adve [14] have derived approximate outage probability results for selection cooperation. These approximations are significantly better than the available highSNR approximations [15]. Here, we derive the exact outage results for all SNR values. If the node,

$$
r_{c}=\underset{r_{c} \in \mathcal{R}(s)}{\arg \max }\left\{\left|h_{r_{c}, d}\right|^{2}\right\}
$$

is selected by the destination for relaying, the mutual information of the selection cooperation scheme is given by

$$
I_{\text {sel }}=\frac{1}{2} \log _{2}\left(1+\operatorname{SNR}\left(\left|h_{s d}\right|^{2}+\left|h_{r_{c} d}\right|^{2}\right)\right) .
$$

By selecting only one cooperative partner, selection cooperation avoids the problem of bandwidth expansion inherent in the repetition-based protocol (the pre-log term is $1 / 2$ compared to $1 /(m+1))$. Conditioned on the decoding set, the outage probability, $\operatorname{Pr}\left[I_{\text {sel }}<R|| \mathcal{R}(s) \mid=1, \ldots, k\right]$, is

$$
\operatorname{Pr}\left(\left|h_{s d}\right|^{2}+\left|h_{r_{c} d}\right|^{2}<\frac{2^{2 R}-1}{\mathrm{SNR}}\right) .
$$

In Appendix $\mathrm{A}$ we have derived the $\mathrm{CDF}$ required to compute (18). Therefore, the exact outage probability of selection 


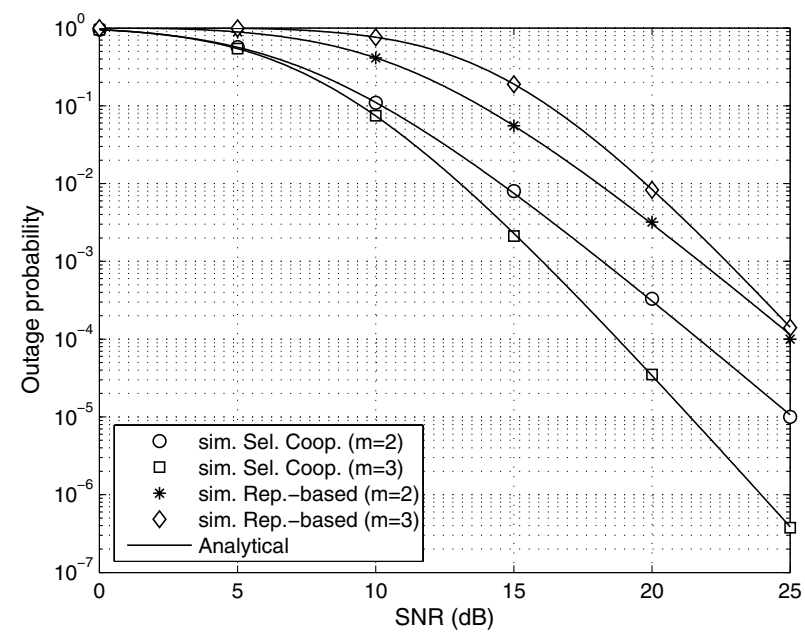

Fig. 2. Outage probability versus SNR in $\mathrm{dB}$ (perfect spectrum acquisition).

cooperation can be expressed as

$$
\begin{aligned}
P_{\text {out }} & =\sum_{i=0}^{m}\left(\begin{array}{c}
m \\
k
\end{array}\right)\left(e^{-\lambda_{s r} \hat{\gamma}}\right)^{i}\left(1-e^{\lambda_{s r} \hat{\gamma}}\right)^{m-i} \\
& \times \lambda_{s d} \sum_{n=0}^{i}(-1)^{n}\left(\begin{array}{c}
i \\
n
\end{array}\right) \frac{e^{-n \lambda_{r d} \hat{\gamma}}-e^{-\lambda_{s d} \hat{\gamma}}}{\lambda_{s d}-n \lambda_{r d}},
\end{aligned}
$$

where $\hat{\gamma}=\frac{2^{2 R}-1}{\mathrm{SNR}}$. As explained for the repetition-based system, the outage probability calculation for selection cooperation under imperfect spectrum acquisition is straightforward.

\section{Simulation Results And Discussion}

In this section, we present simulation results to support our analysis. All curves shown in Figs. $2-4$ correspond to $\lambda_{s d}=4, \lambda_{s r}=2$ and $\lambda_{r d}=1$. In the simulations, we have set $R=1 \mathrm{bit} / \mathrm{sec} / \mathrm{Hz}$. Fig. 2 illustrates the outage probability of repetition-based and selection cooperation schemes under the perfect spectrum acquisition scenario. Clearly, both schemes have the same diversity order as their outage curves show identical slopes in the high SNR regime (see curves corresponding to $m=2$ and 3 ). With $m$ relays helping the source, a diversity order of $m+1$ can be achieved. However, selection cooperation exhibits a better outage performance compared to the repetition-based scheme.

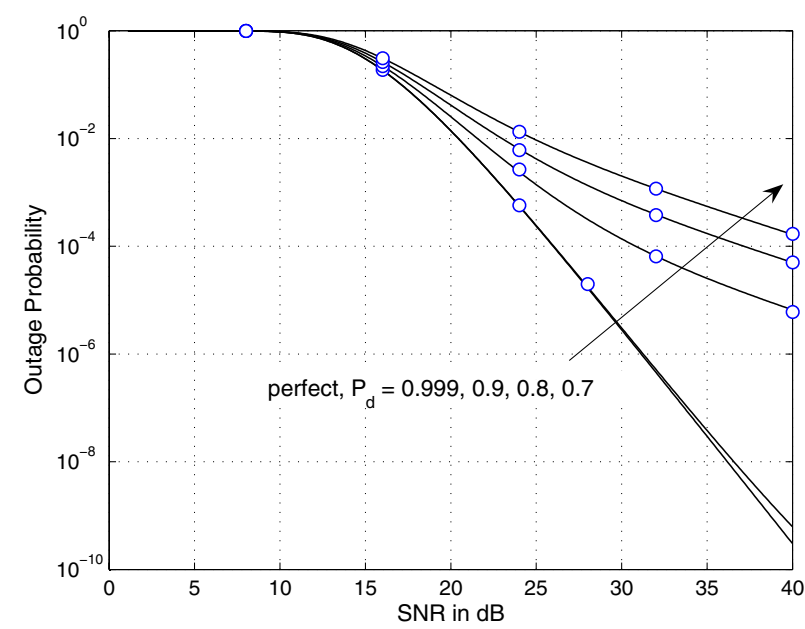

Fig. 3. Outage probability versus SNR in $\mathrm{dB}$ for the repetition-based scheme. Solid lines show theoretical results and dots are for simulations.

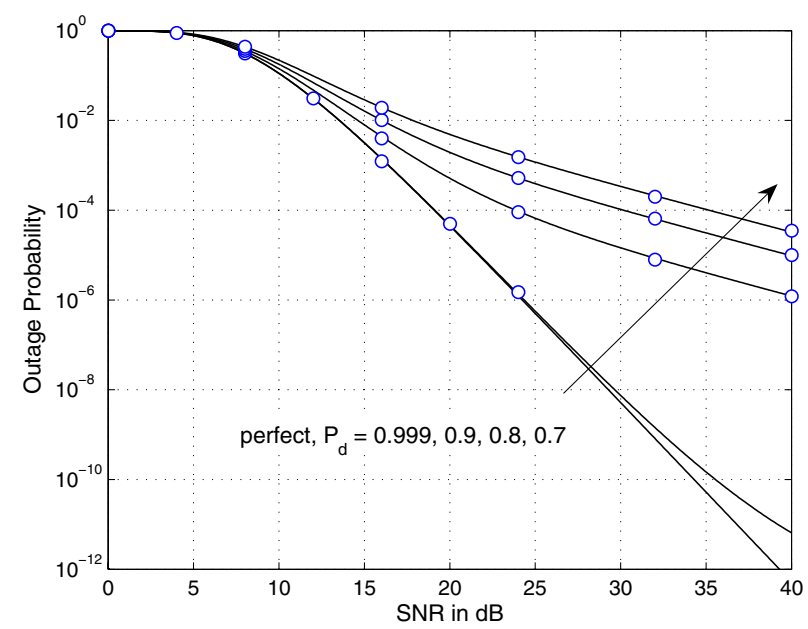

Fig. 4. Outage probability versus SNR in dB for selection cooperation. Solid lines show theoretical results and dots are for simulations.

Figs. 3 and 4 shows the outage probability of the repetitionbased scheme and selection cooperation when spectrum acquisition is not always guaranteed, i.e., $P_{d}<1$. For comparison, we have also plotted the case of perfect spectrum acquisition. Clearly, the outage performance of both schemes deteriorates when there is a chance that the relays are unable to obtain

$$
F_{\mathcal{X}}(x)=\frac{\lambda_{r d}^{k}}{\left(\lambda_{r d}-\lambda_{s d}\right)^{k}}\left(1-e^{-\lambda_{s d} x}\right)-\lambda_{s d} \sum_{j=1}^{k} \frac{\lambda_{r d}^{k-j}}{\left(\lambda_{r d}-\lambda_{s d}\right)^{k-j+1}}\left(1-\left(\sum_{n=0}^{j-1} \frac{\left(\lambda_{r d} x\right)^{n}}{n !}\right) e^{-\lambda_{r d} x}\right)
$$

$$
\begin{aligned}
P_{\text {out }}=\left(1-e^{-\lambda_{s d} \gamma}\right)\left(1-e^{-\lambda_{s r} \gamma}\right)^{m} & +\sum_{i=1}^{m}\left(\begin{array}{c}
m \\
i
\end{array}\right)\left(e^{-\lambda_{s r} \gamma}\right)^{i}\left(1-e^{\lambda_{s r} \gamma}\right)^{m-i}\left(\frac{\lambda_{r d}}{\lambda_{r d}-\lambda_{s d}}\right)^{i} \\
& \left(1-e^{-\lambda_{s d} \gamma}-\lambda_{s d} \sum_{j=1}^{i} \frac{\lambda_{r d}^{-j}}{\left(\lambda_{r d}-\lambda_{s d}\right)^{1-j}}\left(1-\left(\sum_{n=0}^{j-1} \frac{\left(\lambda_{r d} \gamma\right)^{n}}{n !}\right) e^{-\lambda_{r d} \gamma}\right)\right)
\end{aligned}
$$




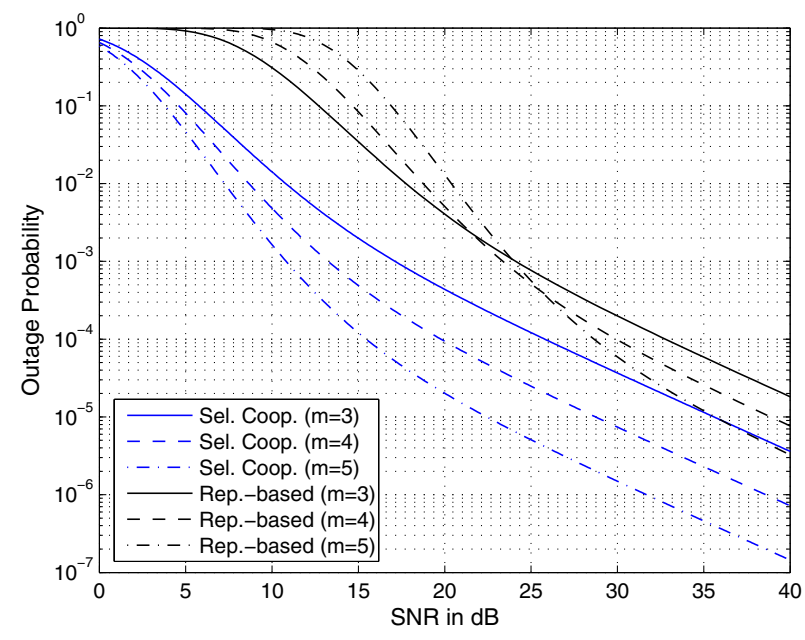

Fig. 5. Comparison of the outage performance for different $m . \lambda_{s d}=1.5$, $\lambda_{s r}=0.5$ and $\lambda_{r d}=0.25$.

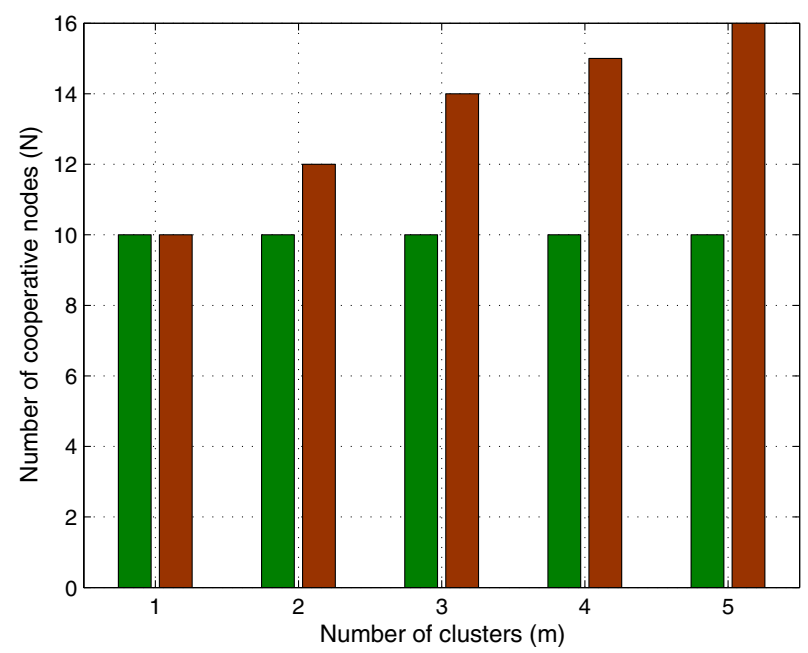

Fig. 6. The necessary number of sensing nodes for different cluster sizes.

spectrum. In Fig. 5 we fix $P_{d}=0.8$, vary $m$ from three to five and make a comparison between the repetition-based scheme and selection cooperation. An interesting observation drawn from Fig. 5 is that using more relays does not necessarily decrease the probability of outage in the case of repetitionbased relaying. Increasing the number of relays tends to increase the number of relays that are able to correctly decode the source signals during phase I. However, due to the time division fashion in which the protocol operates, as well as the ability of the relays to obtain spectrum, increasing numbers of active relays can increase the outage probability. Therefore, an optimal number of relay nodes exists which depends on the SNR, the probability of acquiring spectrum and the channel conditions. On the other hand, in selection cooperation, increasing $m$ always improves the outage performance. However, from a practical point of view, increasing $m$ creates significant signalling overheads for the system. Therefore, as for the repetition-based protocol, a system designer will have to decide on an optimum number of relays in order to limit the implementation complexity.
As described in Section II, one way of improving the spectrum acquisition capability of relays is to employ a cooperative sensing strategy. When more nodes participate, the reliability of finding spectrum increases and leads to better outage performance [7, cf. Fig. 3]. When cooperative spectrum sensing is employed in each cluster, Fig. 6 shows the number of cooperative nodes required to guarantee almost full diversity performance, i.e., near optimum spectrum acquisition performance. For details of this calculation, we refer an interested reader to [7, Eqs. (29), (30)]. In the case of selection cooperation, the same procedure was followed to find the design equation. The parameters considered are from [7, cf. Fig. 4] except $\lambda_{s d}=4, \lambda_{s r}=2$ and $\lambda_{r d}=1.5$. While the number of nodes required to participate in cooperative spectrum sensing for the repetition-based scheme is ten, cooperative selection requires more nodes as $m$ is increased from one to five. Increasing $N$ beyond the numbers shown in Fig. 6 does not significantly improve the outage probability, and near optimum performance is exhibited.

\section{COnClusions}

In this paper, we have considered a cooperative communication system in which the source is assisted by several cognitive relays. The relays opportunistically borrow spectrum from a primary user to transmit information to the destination. Hence, the cooperative gains of this system depend heavily on the ability of the relays to successfully obtain spectrum. We have derived exact expressions for the outage probability of the repetition-based scheme and selection cooperation. When the spectrum acquisition is not always guaranteed, the outage performance of both systems degrades significantly. The outage performance can be improved if cooperative sensing is employed. However, the number of cooperative nodes required for selection cooperation to guarantee near full diversity performance is higher than that of the repetitionbased scheme. The closed-form outage expressions derived have been validated by simulations.

\section{APPENDIX A}

In this Appendix we derive the $\mathrm{CDF}$ of the $\mathrm{RV} \mathcal{Z}$ defined as $\mathcal{Z}=\mathcal{Z}_{1}+\mathcal{Z}_{2}$, where $\mathcal{Z}_{1}$ is exponential with parameter $\lambda_{0}$ and $\mathcal{Z}_{2}=\max \left\{\mathcal{V}_{i}\right\}, \mathcal{V}_{i}$ is exponential with parameter $\lambda_{1}$ and $i=1, \ldots, k$. The $\mathrm{CDF}$ of $\mathcal{Z}_{2}$ is given by

$$
F_{\mathcal{Z}_{2}}(z)=\left(1-e^{-\lambda_{1} z}\right)^{k} .
$$

Rewriting (20) as a sum using the binomial theorem, we obtain

$$
F_{\mathcal{Z}_{2}}(z)=\sum_{n=0}^{k}(-1)^{n}\left(\begin{array}{l}
k \\
n
\end{array}\right) e^{-\lambda_{1} n z} .
$$

Taking into account the independence of $\mathcal{Z}_{1}$ and $\mathcal{Z}_{2}$, the CDF of $\mathcal{Z}$ is $F_{\mathcal{Z}}(z)=\int_{0}^{z} F_{\mathcal{Z}_{2}}(z-x) p_{\mathcal{Z}_{1}}(x) d x$ where $p_{\mathcal{Z}_{1}}(x)$ is the PDF of $\mathcal{Z}_{1}$. Therefore,

$$
F_{\mathcal{Z}}(z)=\lambda_{0} \sum_{n=0}^{k}(-1)^{n}\left(\begin{array}{l}
k \\
n
\end{array}\right) e^{-n \lambda_{1} z} \int_{0}^{z} e^{-\left(\lambda_{0}-n \lambda_{1}\right) x} d x .
$$


Finally, simplifying the integral, we obtain

$$
F_{\mathcal{Z}}(z)=\lambda_{0} \sum_{n=0}^{k}(-1)^{n}\left(\begin{array}{l}
k \\
n
\end{array}\right) \frac{e^{-n \lambda_{1} z}-e^{-\lambda_{0} z}}{\lambda_{0}-n \lambda_{1}} .
$$

\section{APPENDIX B \\ DISTRIBUTED RELAYS}

In the case where the relays are spread over a wide area, the source-relay and relay-destination link strengths will vary. We denote the parameters for relay $i$ by $\lambda_{s r_{i}}$ and $\lambda_{r d_{i}}$. Similarly, the detection probabilities denoted by $P_{i d}$ will vary across the relays. In this scenario, the more general version of (7) is

$$
P_{\text {out }}=\sum_{|\mathcal{R}(s)|=0}^{m} \sum_{\mathcal{R}(s)} \operatorname{Pr}[I<R \mid \mathcal{R}(s)] \operatorname{Pr}[\mathcal{R}(s)],
$$

where the summation over $\mathcal{R}(s)$ is over all subsets of $\{1,2, \ldots, m\}$ of size $|\mathcal{R}(s)|$. When $|\mathcal{R}(s)|=k$, denote the set $\mathcal{R}(s)$ by $\left(q_{1}, q_{2}, \ldots, q_{k}\right)$ and define the new parameters $\mu_{1}, \mu_{2}, \ldots, \mu_{k+1}$ by $\mu_{i}=\lambda_{r_{q_{i}} d}, i=2, \ldots, k+1$ and $\mu_{1}=\lambda_{s d}$. With this notation, (8) can be computed since the sum of exponentials, $Y=\left|h_{s d}\right|^{2}+\sum_{c=1}^{k}\left|h_{r_{c} d}\right|^{2}$, has the CDF [19]

$$
F_{Y}(y)=1-\sum_{j=1}^{m} \prod_{k \neq j}\left(\frac{\mu_{k}}{\mu_{k}-\mu_{j}}\right) e^{-\mu_{j} y}
$$

The remaining probability in (24) is given in [7] as

$$
\operatorname{Pr}[\mathcal{R}(s)]=\prod_{i=1}^{k}\left(e^{-\lambda_{q_{i}} \gamma}\right) \prod_{j=k+1}^{m}\left(1-e^{-\lambda_{\bar{q}_{j}} \gamma}\right),
$$

where $\left(\bar{q}_{k+1}, \ldots, \bar{q}_{m}\right)$ are the relays not in $\mathcal{R}(s)$. Hence, substituting (25) and (26) in (24) gives the outage probability for the repetition-based case.

For the imperfect spectrum acquisition case, (24) and (25) still hold and we require the straightforward extension of (26) given by

$$
\operatorname{Pr}[\mathcal{R}(s)]=\prod_{i=1}^{k}\left(e^{-\lambda_{q_{i}} \gamma} P_{q_{i} d}\right) \prod_{j=k+1}^{m}\left(1-e^{-\lambda_{\bar{q}_{j}} \gamma} P_{\bar{q}_{j} d}\right) .
$$

We note that in [20] Beaulieu and $\mathrm{Hu}$ have also derived a closed-form expression for the outage probability of the repetition-based scheme with distributed relays. However, the derivation given in [20] uses a different approach and does not consider cognitive relays.

For selection cooperation, the outage probability can still be written in the form of (24) with $\operatorname{Pr}[\mathcal{R}(s)]$ given by (26). The remaining probability in (24) is derived below.

$$
\begin{aligned}
\operatorname{Pr}\left[I_{\text {sel }}\right. & <R \mid \mathcal{R}(s)] \\
& =\operatorname{Pr}\left[\left|h_{s d}\right|^{2}+\left|h_{r_{c} d}\right|^{2}<\hat{\gamma}\right] \\
& =\mathbb{E}\left[\prod_{i=1}^{k}\left\{1-e^{-\lambda_{q_{i}}\left(\hat{\gamma}-\left|h_{s d}\right|^{2}\right)}\right\}\right] \\
& =\lambda_{s d} \int_{0}^{\hat{\gamma}}\left(1+\sum_{r=1}^{k}(-1)^{r} e^{-\beta_{r}(\hat{\gamma}-z)}\right) e^{-\lambda_{s d} z} d z,
\end{aligned}
$$

where $\mathbb{E}[\cdot]$ is the expectation operator, $\beta_{r}=\sum_{\mathcal{S}_{r}} \sum_{i \in \mathcal{S}_{r}} \lambda_{q_{i}}$ and the outer summation over $\mathcal{S}_{r}$ is over all $r$-tuples chosen from $\left(q_{1}, q_{2}, \ldots, q_{k}\right)$. Hence, we obtain the final expression

$$
\begin{aligned}
\operatorname{Pr}\left[I_{s e l}<R \mid \mathcal{R}(s)\right] & =1-e^{-\lambda_{s d} \hat{\gamma}} \\
& +\lambda_{s d} \sum_{r=1}^{k}(-1)^{r}\left[\frac{e^{-\beta_{r} \hat{\gamma}}-e^{-\lambda_{s d} \hat{\gamma}}}{\lambda_{s d}-\beta_{r}}\right]
\end{aligned}
$$

A simplified high SNR version of (29) can be found in [15].

\section{ACKNOWLEDGEMENT}

The authors would like to thank Mansoor Shafi and Michael Faulkner for their valuable comments.

\section{REFERENCES}

[1] Federal Communications Commission (FCC), "Facilitating opportunities for flexible, efficient, and reliable spectrum use employing cognitive radio technologies," ET Docket No. 03-108, Mar. 2005.

[2] Cognitive Radio Technology, [Online] Available: http://www.ofcom.org. $\mathrm{uk} /$ research/technology/overview/emer_tech/cograd/cograd_main.pdf

[3] J. Mitola III, Cognitive radio: An integrated agent architecture for software defined radio, Ph.D Thesis, KTH Royal Institute of Technology, Sweden, May 2000.

[4] F. K. Jondral, "Cognitive radio: A communications engineering view," IEEE Wireless Commun. Mag., vol. 14, pp. 28-33, Aug. 2007.

[5] R. Pabst et al., "Relay-based deployment concepts for wireless and mobile broadband radio," IEEE Commun. Mag., vol. 42, pp. 80-89, Sept. 2004.

[6] T. Fujii and Y. Suzuki, "Ad-hoc cognitive radio-Development to frequency sharing system by using multi-hop network," in Proc. IEEE DySPAN 2005, Maryland, MD, Nov. 2005, pp. 589-592.

[7] K. Lee and A. Yener, "Outage performance of cognitive wireless relay networks," in Proc. IEEE GLOBECOM 2006, San Francisco, CA, Nov. 2006.

[8] J. N. Laneman and G. W. Wornell, "Distributed space-time-coded protocols for exploiting cooperative diversity in wireless networks," IEEE Trans. Inform. Theory, vol. 49, pp. 2415-2425, Oct. 2003.

[9] K. Hamdi and K. B. Letaief, "Cooperative communications for cognitive radio networks," in Proc. PGNet 2007, Liverpool, UK, June 2007.

[10] J. Mietzner, L. Lampe and R. Schober, "Distributed transmit power allocation for relay-assisted cognitive-radio systems," in Proc. Asilomar Conf. Signals, Systems, and Computers (ACSSC 2007), Pacific Grove, CA, Nov. 2007.

[11] Y. Zhao, R. S. Adve and T. J. Lim, "Outage probability at arbitrary SNR in cooperative diversity networks," IEEE Commun. Lett., vol. 9, pp. 700-702, Aug. 2005.

[12] H. A. Suraweera, P. J. Smith and J. Armstrong, "Outage probability of cooperative relay networks in Nakagami- $m$ fading channels," IEEE Commun. Lett., vol. 10, pp. 834-836, Dec. 2006.

[13] A. Bletsas, A. Khisti, D. Reed and A. Lippman, "A simple cooperative diversity method based on network path selection," IEEE J. Sel. Areas Commun., vol. 24, pp. 659672, Mar. 2006.

[14] E. Beres and R. S. Adve, "Outage probability of selection cooperation in the low to medium SNR regime," IEEE Commun. Lett., July 2007.

[15] E. Beres and R. S. Adve, "On selection cooperation in distributed networks," IEEE Trans. Wireless Commun., Accepted. [Online] http: //www.comm.utoronto.ca/ eberes/Selection.pdf

[16] A. Ghasemi and E. S. Sousa, "Collaborative spectrum sensing for opportunistic access in fading environments," in Proc. IEEE DySPAN 2005, Baltimore, MD, Nov. 2005, pp. 131-136.

[17] C. A. Coelho, "The generalized integer Gamma distribution-A basis for distributions in multivariate statistics," J. Multivariate Anal., vol. 64, pp. 86-102, 1998

[18] I. S. Gradshteyn and I. M. Ryzhik, Table of Integrals, Series and Products. 6th ed., San Diego: CA, Academic Press, 2000.

[19] N. L. Johnson and S. Kotz, Continuous Univariate Distributions. vol. II, New York: John Wiley, 1970.

[20] N. C. Beaulieu and J. Hu, "A closed-form expression for the outage probability of decode-and-forward relaying in dissimilar Rayleigh fading channels," IEEE Commun. Lett., vol. 10, pp. 813-815, Dec. 2006. 